[0212-7199 (2003) 20: 12; pp 617-620] ANALES DE MEDICINA INTERNA Copyright (C) 2003 ARAN EDICIONES, S.L.

AN. MED INTERNA (Madrid) Vol. 20, N. ${ }^{\circ} 12$, pp. 617-620, 2003

\title{
Sarcoidosis en el área sanitaria de León. Epidemiología y clínica
}

\author{
M. ALCOBA LEZA, M. R. PÉREZ-SIMÓN, J. M. GUERRA LASO, J. A. CARRO \\ FERNÁNDEZ, M. A. ALONSO FERNÁNDEZ, B. BLANCO IGLESIAS, P. BUENO \\ SUÁREZ, I. MUINELO VOCES
}

Servicio de Medicina Interna II. Hospital de León. León

SARCOIDOSIS IN A SANITARY AREA AT LEON (SPAIN). EPIDEMIO-
LOGY AND CLINICAL FEATURES

\section{RESUMEN}

Objetivos: La incidencia de la sarcoidosis difiere de unas zonas geográficas a otras, atribuyéndose las diferencias, entre otros factores, a variaciones climáticas. En nuestro país hay un número limitado de estudios que relacionan los casos observados con una población determinada. El objetivo del presente trabajo ha sido estudiar la epidemiología de la sarcoidosis en el área sanitaria de León, comparándola con la de otras zonas geográficas de nuestro país. Adicionalmente se describen las características clínico-radiológicas de los casos observados.

Material y método: Se analizan los casos de sarcoidosis asistidos en el área en el periodo 1993-2001, estudiando la incidencia, edad, sexo, hábitat y características clínico-radiológicas. Para la incidencia se han utilizado los datos de población del Insalud referidos al área. Para el clima se ha contado con la información facilitada por el Instituto Nacional de Meteorología.

Resultados: La incidencia en el área fue de 1,37/100.000 habitantes/año, similar a la encontrada en Cataluña, Galicia y otras provincias de la comunidad de Castilla y León. Hay más casos en el sexo femenino debido al mayor número de mujeres a partir de los 40 años. No hay diferencias en la incidencia entre las zonas urbanas y rurales del área. La clínica no difiere de lo descrito en otras zonas de nuestro país.

Conclusión: Aunque existen diferencias climáticas entre distintas zonas geográficas de España, probablemente no son suficientemente acusadas para que ello se refleje en la incidencia de la sarcoidosis, que es similar en todas ellas. Esto es válido al menos para la mitad norte de la península.

PALABRAS CLAVE: Sarcoidosis. Incidencia. Epidemiología.

\section{ABSTRACT}

Background: The incidence of sarcoidosis is different among distincts geographic areas, probably due to climate variations. At present, there is in Spain few studies that correlate the observed cases with a concrete population. The aim of this study is to investigate the epidemiology of sarcoidosis in Leon's sanitary area, in comparison with others spanish geographic areas. Moreover, we present a description of the clinic and radiologic characteristics of the studied cases.

Method: We analyzed the cases of sarcoidosis attended in the area from 1993 until 2001. The incidence, age, sex, place of residence and clinic-radiologic characteristics are described. The population data of the sanitary area to calculate the incidence were obtain from the Insalud's data base. The climatology values were collected from the National Institute of Meteorology.

Results: The incidence of sarcoidosis in Leon's sanitary area was 1,37/100.000 habitants/year, and was similar to that founded in Catalonia, Galizia and others provinces of the Castilla and Leon community. We observed a higher incidence of sarcoidosis in female patients, because of predominance of cases among women aged forty years or more. No differences between rural and urban populations were founded. The clinical characteristics were similar those described in other geographics areas of our country.

Conclusion: Although there are climate differences between the geographics areas of Spain, these aren't enough to explain his influence in the incidence of sarcoidosis, which is similar in all of them, at least in the middle north of the country.

KEY WORDS: Sarcoidosis. Incidence. Epidemiology.

Alcoba Leza M, Pérez-Simón MR, Guerra Laso JM, Carro Fernández JA, Alonso Fernández MA, Blanco Iglesias B, Bueno Suárez P, Muinelo Voces I. Sarcoidosis en el área sanitaria de León. Epidemiología y clínica. An Med Interna (Madrid) 2003; 20: 617-620.

\section{INTRODUCCIÓN}

La sarcoidosis es una enfermedad de distribución universal. Su incidencia y prevalencia varían de unas zonas geográficas a otras (1-4) e incluso en un mismo país se aprecian diferencias entre sus regiones (1). Estas diferencias se han atribuido, entre otros factores, a variaciones climáticas, dándose más la enfermedad en climas fríos $(1,5)$. La incidencia y prevalencia cambian también según el método empleado en la recogida de casos $(2,6)$, lo que ha de tenerse en cuenta al analizar las diferencias halladas. En nuestro país han aparecido en los últimos 20 años diversas publicaciones sobre sarcoidosis que recogen casos diagnosticados en los centros hospitalarios, en general pertenecientes a la mitad norte de la península (714). Algunos trabajos relacionan los casos estudiados con una población determinada $(8,10-12)$, lo que permite hacer una estimación de la incidencia en el área geográfica correspondiente.

Trabajo aceptado: 11 de junio de 2003 
León es una provincia de la meseta norte que se caracteriza desde el punto de vista climático por temperaturas en general frías durante el invierno y veranos frescos. Resulta interesante por ello investigar la incidencia de la sarcoidosis en esta provincia comparándola con la encontrada en otras zonas de nuestro país de clima similar o diferente. El objetivo principal de este trabajo ha sido estudiar desde el punto de vista epidemiológico la sarcoidosis en el área sanitaria de León. Adicionalmente se describen también las características clínicoradiológicas de los casos estudiados.

\section{MATERIAL Y MÉTODOS}

El Hospital de León es un complejo hospitalario de 805 camas al que desde 1991 corresponde la asistencia del área sanitaria de León que, según los datos del Insalud de 1999 abarca una población de 366.385 habitantes de los que 172.898 corresponden a zonas urbanas (León capital, La Bañeza y Astorga) y 193.487 a municipios rurales. Desde 1993 existe en el archivo del hospital un sistema de codificación a través del cual se solicitaron todas las historias clínicas de enfermos dados de alta entre el 1-1-1993 y el 31-12-2001 con el diagnóstico de sarcoidosis. Se aceptó el diagnóstico de sarcoidosis si se daban uno de estos dos criterios: a) clínica compatible junto a la existencia de un estudio histológico con presencia de granulomas no caseificantes y exclusión de otras enfermedades causantes de este tipo de granulomas; y b) presencia de adenopatías hiliares en pacientes con eritema nudoso y evolución espontánea favorable con un seguimiento mínimo de seis meses. En estos pacientes se consideró también la existencia de una reacción de Mantoux negativa y la elevación de la enzima de conversión de la angiotensina (ECA).

En cada caso se recogieron los siguientes datos: edad, sexo, cuadro clínico, hábitat y resultado de los estudios de diagnóstico por imagen (radiografía simple de tórax, TAC torácico, y Gammagrafía con ${ }^{67} \mathrm{Ga}$ ).

En el estudio de la incidencia se ha utilizado en el denominador la población del área sanitaria según los datos del Insalud de 1999.

Los valores climáticos son los que constan en la información facilitada por el Instituto Nacional de Meteorología (INM) (15), reproduciéndose con autorización expresa del mismo.

\section{RESULTADOS}

Se revisaron 62 historias en las que en el informe de alta constaba el diagnóstico de sarcoidosis. Se desecharon 11 porque el diagnóstico se había hecho con anterioridad al 1-01-93 y cinco porque no reunían ninguno de los criterios exigidos. De los 46 pacientes restantes, 34 se diagnosticaron con estudio histológico y 12 con arreglo al criterio clínico-radiológico. La incidencia fue de 1,37/100.000 habitantes/ año.

Hábitat. En el medio rural vivían 23 pacientes y en el urbano otros 23. Con arreglo a la población de uno y otro medio la incidencia fue de 1,32 y 1,47/100.000 habitantes/año respectivamente.

Edad y sexo. Se reflejan en el histograma de la figura 1. Existe un predominio de mujeres (relación mujer/varón

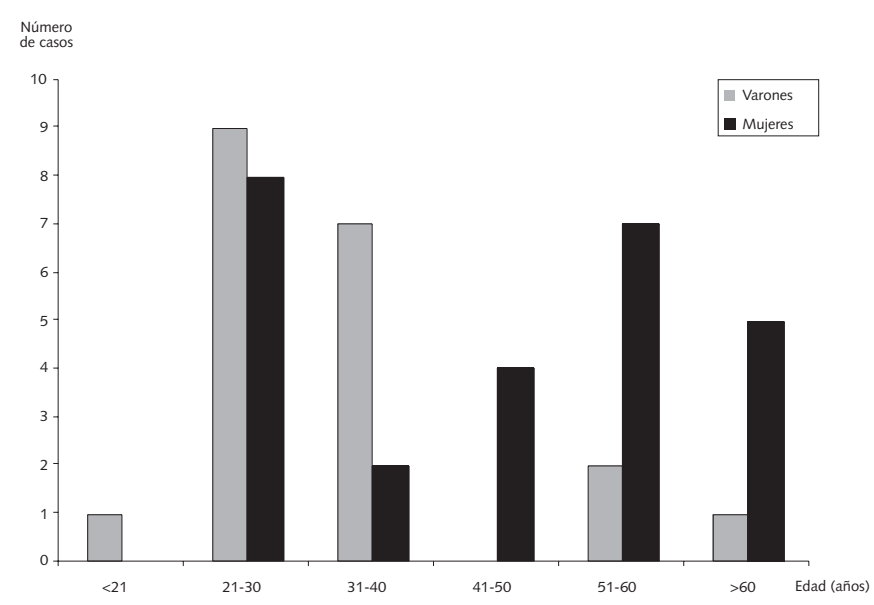

Fig. 1. Frecuencia por edades según sexos.

$26 / 20=1,35)$ Sin embargo, si se distribuyen los casos en dos grupos, uno formado por los mayores de 40 años y otro por los que tienen $\leq 40$ años, la relación varía. Así en el primer grupo la relación mujer/varón es de 16/3=5,66 mientras que en el segundo es de 10/17=0,58.

Clínica. Las manifestaciones clínicas quedan reflejadas en la tabla I. Como puede apreciarse casi la mitad de los pacientes cursaron con síntomas respiratorios y eritema nudoso, siendo también destacable la frecuencia con que aquejaban síntomas constitucionales y artralgias o artritis.

\begin{tabular}{lc}
\multicolumn{2}{c}{ TABLA I } \\
\multicolumn{2}{c}{ CLÍNICA } \\
\hline Manifestaciones clínicas & $\begin{array}{c}\text { Número de } \\
\text { pacientes (\%) }\end{array}$ \\
\hline Asintomático & $8(17 \%)$ \\
Síntomas respiratorios & $20(43 \%)$ \\
Eritema Nudoso & $19(41 \%)$ \\
Síntomas constitucionales* & $14(30 \%)$ \\
Artralgias/artritis & $12(20 \%)$ \\
Fiebre* & $8(17 \%)$ \\
Afectación cutánea*** & $2(4 \%)$ \\
Adenopatías periféricas & $2(4 \%)$ \\
Hipercalcemia & $2(4 \%)$ \\
Parotiditis & $1(2 \%)$ \\
\hline
\end{tabular}

*: Astenia, anorexia y pérdida de peso.
**: En cuatro casos asociada a síntomas constitucionales.
***: Exceptuando eritema nudoso.

Diagnóstico por imagen. El estadiaje de los enfermos con arreglo a los hallazgos de la radiografía simple de tórax se expone en la tabla II. Se realizó TAC torácico a 41 pacientes siendo los resultados concordantes con la radiografía en 39 $(95 \%)$ y no concordantes en dos: uno en que la radiografía simple se interpretó como normal y en la TAC se encontraron adenopatías en el tórax y otro considerado en estadio I en la radiografía simple y en el que el TAC mostró también patología pulmonar. 


\begin{tabular}{cc}
\multicolumn{3}{c}{ TABLA II } \\
& ESTADIAJE \\
\hline Estadio & Número de \\
& casos (\%) \\
\hline 0 & $2(4 \%)$ \\
I & $34(74 \%)$ \\
II & $6(13 \%)$ \\
III & $4(9 \%)$ \\
\hline
\end{tabular}

Se practicó gammagrafía con ${ }^{67} \mathrm{Ga}$ en 32 pacientes. En todos ellos se encontraron hallazgos patológicos en el tórax, en general concordantes con la radiología simple: sólo en un caso en que ésta se consideró normal, se apreció hipercaptación en adenopatías hiliares y parénquima pulmonar. Siete casos $(22 \%)$ presentaban hipercaptación en zonas extratorácicas: cuatro en parótidas, uno en nódulos subcutáneos, uno en adenopatías periféricas y uno en adenopatías abdominales.

\section{DISCUSIÓN}

Este estudio tiene dos limitaciones: es retrospectivo y, por otro lado, los casos se han recogido exclusivamente entre los diagnosticados en el hospital del área. Por ello es muy proba- ble que la incidencia hallada sea inferior a la real. En el estudio realizado en la isla de Man (6) durante el periodo 19771983 de modo prospectivo e implicando en la recogida de casos a los médicos generales, la incidencia fue significativamente mayor que en los 15 años previos en que los casos no se recogieron en aquellas condiciones. Sin embargo, es posible comparar la incidencia encontrada en el área sanitaria de León con la de otras zonas de nuestro país pues aquellos estudios que relacionan el número de casos con una población dada $(8,10-12)$ fueron realizados con una metodología similar, esto es, de modo retrospectivo y a partir de los casos diagnosticados en los hospitales. Los datos de estos estudios se exponen en la tabla III. Como puede observarse la incidencia en el área sanitaria de León es similar no sólo a la hallada en otras provincias de la meseta sino también en zonas costeras, como Cataluña o Galicia. Existen variaciones climáticas entre unas provincias y otras, como queda reflejado en la tabla IV que reproduce la información proporcionada por el INM; como puede apreciarse la temperatura media es inferior en el invierno en las provincias de la meseta en comparación con las regiones costeras. Cabría esperar por ello que la incidencia fuera mayor en las primeras y, sin embargo, es sensiblemente igual en todas ellas (si se exceptúa Palencia en que es algo superior, aunque debe considerarse que el periodo de tiempo analizado es breve y el número total de casos pequeño). Probablemente aquellas diferencias climáticas, aún existiendo, no son suficientemente acusadas para influir de modo decisivo en

\begin{tabular}{|c|c|c|c|c|c|}
\hline \multicolumn{6}{|c|}{ TABLA III } \\
\hline \multicolumn{6}{|c|}{ INCIDENCIA COMPARATIVA } \\
\hline Referencia & $\begin{array}{c}\text { Área } \\
\text { geográfica }\end{array}$ & Periodo & $\begin{array}{l}\text { Número de } \\
\text { habitantes }\end{array}$ & $\begin{array}{c}\text { Número de } \\
\text { casos }\end{array}$ & Incidencia* \\
\hline Fité (12) & Cataluña & $1986-88$ & 6.000 .000 & 245 & 1,32 \\
\hline García Martín (18) & La Coruña & $1974-82$ & 500.000 & 40 & 1,2 \\
\hline Pérez Arellano (10) & Salamanca & $1983-88$ & 366.000 & 30 & 1,4 \\
\hline Villarroya (11) & Palencia & $1988-90$ & 200.000 & 14 & 2,3 \\
\hline Autores & León & $1993-2001$ & 366.385 & 46 & 1,37 \\
\hline
\end{tabular}

*: Casos/100.000 habitantes/año.

TABLA IV

TEMPERATURA MEDIA MENSUAL $\left({ }^{\circ} \mathrm{C}\right)$

\begin{tabular}{|c|c|c|c|c|c|}
\hline Mes & Barcelona & León & Salamanca & Coruña & Palencia \\
\hline Enero & 8,8 & 3,1 & 3,7 & 10,2 & 4,2 \\
\hline Febrero & 9,6 & 4,5 & 5,4 & 10,5 & 5,6 \\
\hline Marzo & 11,1 & 6,6 & 7,3 & 11,3 & 7,4 \\
\hline Abril & 12,9 & 8,5 & 9,6 & 12,1 & 9,4 \\
\hline Mayo & 15,9 & 12,1 & 13,4 & 14,1 & 13,1 \\
\hline Junio & 19,7 & 16,4 & 17,8 & 16,4 & 17,1 \\
\hline Julio & 22,8 & 19,6 & 21,0 & 18,4 & 20,4 \\
\hline Agosto & 23,0 & 19,1 & 20,3 & 18,8 & 20,3 \\
\hline Septiembre & 21,1 & 16,8 & 17,5 & 18,1 & 18,0 \\
\hline Octubre & 17,1 & 11,8 & 12,3 & 15,7 & 13,1 \\
\hline Noviembre & 12,5 & 6,8 & 7,0 & 12,6 & 7,6 \\
\hline Diciembre & 9,7 & 3,8 & 4,1 & 10,9 & 4,4 \\
\hline
\end{tabular}

Barcelona: Aeropuerto del Prat. León: Base aérea de la Virgen del Camino. Salamanca: Base aérea de Matacán.

Fuente: Instituto Nacional de Meteorología (15), con autorización. 
la incidencia. Así los datos de la tabla IV permiten clasificar a todas las provincias, con arreglo al Atlas de Oxford (16), dentro de la categoría de inviernos templados (temperatura media del mes más frío entre $2^{\circ}$ y $13^{\circ}$ ). En cuanto al verano, León y La Coruña entrarían en la categoría de veranos frescos (temperatura media del mes más caluroso entre $10^{\circ}$ y $20^{\circ}$ ) y las demás en la de veranos calurosos, aunque las diferencias entre todas ellas sean inferiores a dos grados si se exceptúa Barcelona cuya temperatura media en el mes más caluroso (agosto) es ligeramente superior a cuatro grados con relación a la que tiene el verano más fresco (La Coruña).

Las notables diferencias halladas tanto en la prevalencia como en la incidencia entre los países del norte y el sur de Europa (1-3) vendrían dadas porque las variaciones climáticas entre aquéllos y estos son mucho más acusadas, como puede apreciarse en el mapa de Hosoda (1) que relaciona el clima con la prevalencia de la enfermedad.

En relación con el hábitat no hemos encontrado el predominio de casos en el medio rural descrito por Pérez Arellano et al (10) en Salamanca ni en términos absolutos ni referido a la población de uno u otro medio.

La sarcoidosis es más frecuente en el sexo femenino según se recoge en la mayoría de las publicaciones, aunque hay algunas excepciones a esta regla $(17,18)$. En la presente serie existe también un predominio femenino. Sin embargo, según nuestros datos, esto es debido al mayor número de mujeres que sufren la enfermedad después de los 40 años, lo que concuerda con los resultados de series muy amplias en las que consta el sexo en los diferentes grupos de edad $(3,5,9)$.

Las características clínicas de los pacientes son similares a las descritas en otras publicaciones de nuestro país. Así, en las que contienen mayor número de casos y la descripción es suficientemente detallada, se cita la existencia de eritema nudoso entre el 40 y el $50 \%(7,9,12)$. Otro tanto cabe decir de la distribución de los casos según el estadio, predominando en casi todos los estudios el estadio I seguido en proporción inferior del II y suponiendo entre ambos más del $70 \%$ de los casos en todas las series (7-13).

Cabe concluir por tanto que aunque existen diferencias climáticas entre distintas regiones en nuestro país la incidencia de la sarcoidosis es similar en todas ellas. Probablemente aquellas diferencias son poco acusadas y ello hace que su influencia sobre la incidencia sea mínima. Esto es así al menos en las regiones de la mitad norte de la península. No disponemos de datos de las regiones del sur que nos permitan generalizar esta afirmación a todo el país.

\section{Bibliografía}

1. Hosoda Y, Yamaguchi M, Hiraga Y. Global epidemiology of sarcoidosis. What story do prevalence and incidence tell us? Clin Chest Med 1997; 18: 681-694.

2. Kitaichi M. Prevalence of sarcoidosis around the world. Sarcoidosis Vasc Diffuse Lung Dis 1998; 15: 16-18.

3. Hillerdal G, Nöu E, Osterman K, Schmekel B. Sarcoidosis: epidemiology and prognosis. A 15-year european study. Am Rev Respir Dis 1984; 130: 29-32.

4. Arribas Castrillo JM. Sarcoidosis. An Med Interna (Madrid) 2000; 17: 513-516.

5. Yamaguchi M, Hosoda Y, Sasaki R, Aoki K. Epidemiological study on sarcoidosis in Japan. Recent trends in incidence and prevalence rates and changes in epidemiological features. Sarcoidosis 1989; 6: 138-146.

6. Parkes SA, Baker SB, Bourdillon RE, Murray CRH, Rakshit M, Sarkies JW et al. Incidence of sarcoidosis in the Isle the Man. Thorax 1985; 40: 284-287.

7. Lorente JA, Peña JM, Barbado FJ, Serrano JA, Fonseca E, García-Alegría J, et al. Perfil clínico-biológico de la sarcoidosis. Análisis de 35 casos. Rev Clin Esp1985; 176: 112-117.

8. García Martín C, Masa JF, Verea H, Fontán J, Mosquera JA, Galdo F, Vázquez Iglesias JL, Pedreira JD, Diz-Losi F. Sarcoidosis. Aspectos epidemiológicos, clínicos y diagnósticos en 40 pacientes. Rev Clin Esp 1985; 176: 32-34.

9. Badrinas F, Morera J, Fité E, Mañá J, Vidal R, Ruiz Manzano J, et al. Sarcoidosis en Cataluña: análisis de 425 casos. Med Clin (Barc) 1989;
93: 81-87.

10. Pérez Arellano JL, Ramos Guevara RM, López Ochoa FJ, Alcázar Montero MC, De León Gómez B, Flores MT. Sarcoidosis. Estudio retrospectivo de 30 casos en Salamanca y comparación con otras series nacionales. Rev Clin Esp 1991; 189: 159-162.

11. Villarroya Lequericaonandía ME, Fernández Jorge MA, Ibáñez Gómez J, Arizcun Morate A, Alonso Mallo E, Pérez Sicilia M. Aten Primaria 1992; 9: 436-438.

12. Fité E, Alsina JM, Mañá J, Pujol R, Ruiz J, Morera J. Epidemiology of sarcoidosis on Catalonia: 1979-1989. Sarcoidosis Vasc Diffuse Lung Dis 1996; 13: 153-158.

13. Castañé J, Rodríguez-Carballeira M, Ochoa A, Esteban L, MuñozRodríguez FJ. Tratamiento y pronóstico de la sarcoidosis. A propósito de 53 casos. An Med Interna (Madrid) 2000; 17: 517-520.

14. Pérez de Llano LA, Romero de Araujo D, Soilán del Cerro JL, Ortíz Piquer M, González-Rosón Fernández O, Gago Hermida D. Sarcoidosis nodular pulmonar, ¿una forma radiológica de presentación infrecuente o una vasculitis granulomatosa? Descripción de tres casos. An Med Interna (Madrid) 2000; 17: 88-91.

15. www.inm.es

16. Cohen SB. Oxford World Atlas. Oxford University Press, Oxford, 1973.

17. Thrasher DR, Briggs DD Jr. Pulmonary Sarcoidosis. Clin Chest Med 1982; 3: 537-563.

18. Gupta SK, Mitra K, Chatterjee S, Chakravarty SC. Br J Dis Chest 1985; 79: $275-283$. 